Highly Energetic Physical Processes and

Mechanisms for Emission from Astrophysical Plasmas

IAU Symposium, Vol. 195, 2000

P. C. H. Martens, S. Tsuruta, and M. A. Weber, eds.

\title{
Recent Satellite Observations of X-ray Emission from AGN
}

\author{
H. Kunieda ${ }^{1}$ \\ Department of Physics, Nagoya University Furo-cho, Nagoya 464-8602, \\ Japan
}

\begin{abstract}
Recent X-ray satellite observations provide plenty of spectral information with high resolution in a broad band. Major results from the spectral observations are related to the interaction of the power-law continuum flux with the ambient plasmas and accretion disks. The most prominent result is the broad iron-line feature from Seyfert I galaxies which is interpreted as the emission from the relativistic accretion disk. Doppler shift and boosting, as well as gravitational redshifts, are considerable at the distance of several Schwarzschild radii from the central black hole. Strong radiation from the central engine ionizes the ambient material of AGN. Edge structure found at around $0.8 \mathrm{keV}$ is attributed to highly ionized oxygen of O VII and O viII. Some iron emission lines from Seyfert II galaxies are emitted by highly photoionized plasmas. The study of fast temporal and spectral variability is the key approach to examine the emission mechanism and structure of the central vicinity of AGN. In 2000, two X-ray observatories (Chandra and XMM will be in orbit, and they will provide us with deeper insights of the physics around the black holes and of the physics of hot plasmas.
\end{abstract}

\section{Current X-ray Missions}

X-ray astronomy satellites in this decade are summarized in Figure 1 together with those in the near future. Bars with gray color indicate nonimaging, largearea detectors with collimators, such as Ginga, XTE, and some instruments of SAX. Missions shown with solid lines are those with telescopes, which drastically enhance the detection sensitivity. Among those telescope missions, the effective area of ROSAT is limited below $2 \mathrm{keV}$, while BBXRT, ASCA, and SAX can cover X-rays up to $10 \mathrm{keV}$, including Fe-K lines at $6.4-6.7 \mathrm{keV}$. This has been made possible by introducing high-throughput X-ray telescopes.

Another advantage of current missions is the energy resolution, especially with focal-plane detectors. Gas-scintillation proportional counters have twice better resolution than those of ordinary proportional counters. The CCD onboard ASCA has one order better energy resolution $E / \mathrm{d} E$ of about 60 at $6 \mathrm{keV}$.

\footnotetext{
${ }^{1}$ Also at: Institute of Space and Astronautical Science, Yoshinodai, Sagamihara, 229-8510, Japan
} 


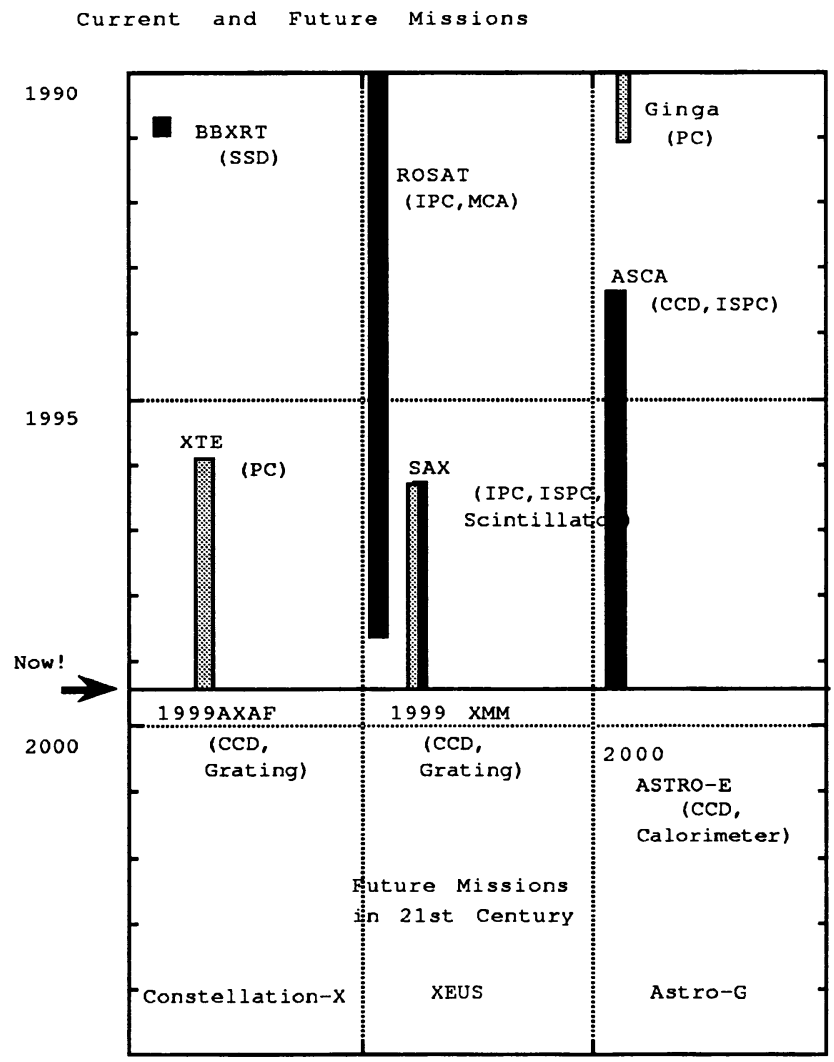

Figure 1. Current and future X-ray missions. (Astro-E should be listed at 2004-2005 instead of 2000.)

\section{AGN Science}

By utilizing better sensitivity in a broad energy band and high resolution spectroscopy, we are able to examine the science of AGN, as shown below. Among various subjects, three important issues are discussed in this paper: the broad iron-line profile, photoionized plasmas, and the link to other galaxies.

\subsection{Broad Fe-Line Profile}

Discovery of the Broad Feature with ASCA In the composite spectrum of Seyfert galaxies observed by Ginga, a line feature was found at $6.4 \mathrm{keV}$, and an enhancement was seen above $10 \mathrm{keV}$ (Nandra \& Pounds 1993). The former is the fluorescence iron- $\mathrm{K}$ line from cold matter illuminated by the central engine. The latter is due to the albedo of thick walls. Before the launch of ASCA, we expected a bright and narrow peak with ten times better energy resolution than ASCA. In the early phase of ASCA operations, we were puzzled by the 
very small line intensity with three times smaller equivalent width than Ginga results when they are fitted with a narrow-line model. One year after, based on better understandings of detector systems, we could report that the iron line from the Seyfert I galaxy MCG-6-30-15 is really broad (Tanaka et al. 1995). The iron line is not missing but is broader than $1 \mathrm{keV}$ (Figure 2a).

Gravitational Red Shift? This is characterized by a peak at $6.4 \mathrm{keV}$ with a tail on the low-energy side. This type of skewed line feature had been considered with the model of a relativistic accretion disk around a black hole. The iron line is the fluorescence from the accretion disk with relativistic speed (Figure 3 ). The blue peak of the double horns of the Doppler effect is boosted in the relativistic case. At the same time, a strong gravitational field causes the red wing. Different inclination angles and disk sizes provide different line features. Observed redshifts suggest the innermost part of the disk approaches to the black hole as close as several Schwarzschild radii.

In Figure 4, line shapes are plotted for the components contributed by different annuli. The innermost part $\left(r<10 R_{\mathrm{s}}\right)$ gives a broader and lower energy $(E<6 \mathrm{keV})$ component (dot-dashed line). Therefore, the line profile change is the key of the disk structure. In the following subsections, we discuss the dependence of the line profile on the central luminosity and on the continuum spectrum.

Luminosity Dependence These broad features were commonly found in most Seyfert I galaxies and similar objects (Nandra et al. 1997). Authors sorted those samples with intrinsic luminosity (Figure 2). Below $10^{45} \mathrm{erg} / \mathrm{sec}$, line features are very similar to the typical ones. On the other hand, those more luminous than $10^{45} \mathrm{erg} / \mathrm{sec}$ show a little higher-energy line feature without red wings, and then no line feature is found in the brightest $\left(L_{\mathrm{X}}>10^{46} \mathrm{erg} / \mathrm{sec}\right)$ group. Authors explained these results by the lack of an inner part of the disk due to the strong radiation field. The innermost part is fully ionized, and the next annulus is highly ionized to produce $6.7 \mathrm{keV}$ lines (Figure $5 \mathrm{~b}$ ).

Intensity Variation The next step of investigation is the line profile change according to the intensity and spectral changes of individual sources. From MCG-6-30-15, Iwasawa reported that the line profile at the flare peak seems to be just the red wing without any $6.4 \mathrm{keV}$ peak (Iwasawa et al. 1999). Just after the flare, the intensity dropped down to $50 \%$, and the line feature returned to the normal broad feature. In this case, more accretion flow came into radii smaller than three Schwarzschild radii, for example, and then the observed profile was dominated by the strongly redshifted component temporally (Figure 5c).

Spectral Change in Mkn 841 Another example, this time from Mkn 841, is the profile change accompanied by the spectral change without intensity change (Misaki et al. 2000). Though the intensity is almost the same within $5 \%$, the power-law slope changed from 1.9 to 1.6. The line profile in the softer state is in a very typical shape, while it becomes only a narrow component in the hard state. Such a line profile without a red wing can not be explained by the lack of an inner part of the disk, like the flare of MCG-6-30-15. An alternative interpretation is the change of the flux distribution of iron lines. More illumination at larger radii 


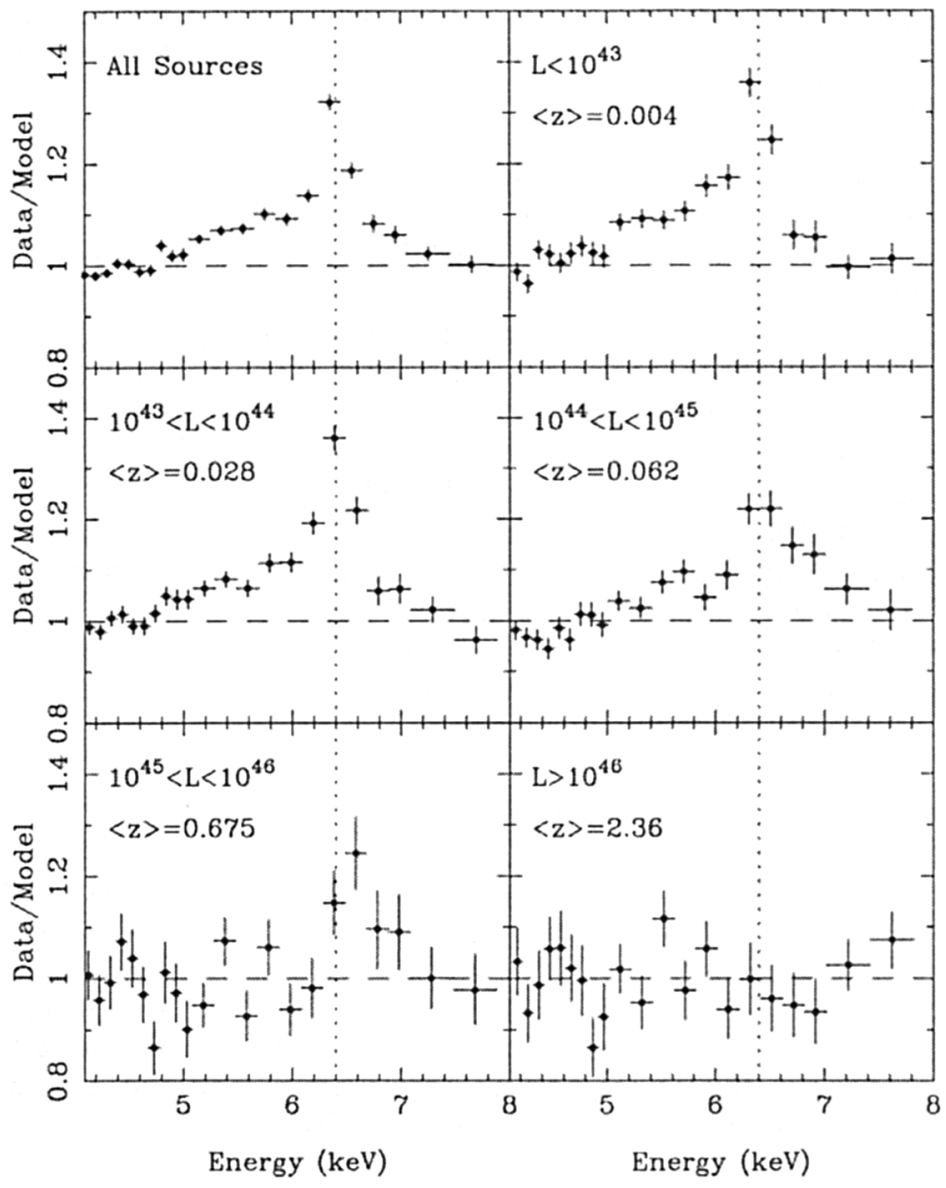

Figure 2. Broad iron-line profile of Seyfert galaxies with different luminosities (Nandra et al. 1998). Ratio of observed spectrum to a single power-law continuum is calculated for Seyferts in the following luminosity levels. (a) Composite line profile of all sample sources, (b) averaged line profile of Seyferts of X-ray luminosity $L_{\mathrm{X}}<10^{43} \mathrm{erg} / \mathrm{s}$, (c) those of $10^{43}<L_{\mathrm{X}}<10^{44} \mathrm{erg} / \mathrm{sec}$, (d) those of $10^{44}<L_{\mathrm{X}}<$ $10^{45} \mathrm{erg} / \mathrm{sec}$, (e) those of $10^{45}<L_{\mathrm{X}}<10^{46} \mathrm{erg} / \mathrm{sec}$, and (f) those of $L_{\mathrm{X}}>10^{46} \mathrm{erg} / \mathrm{sec}$. 


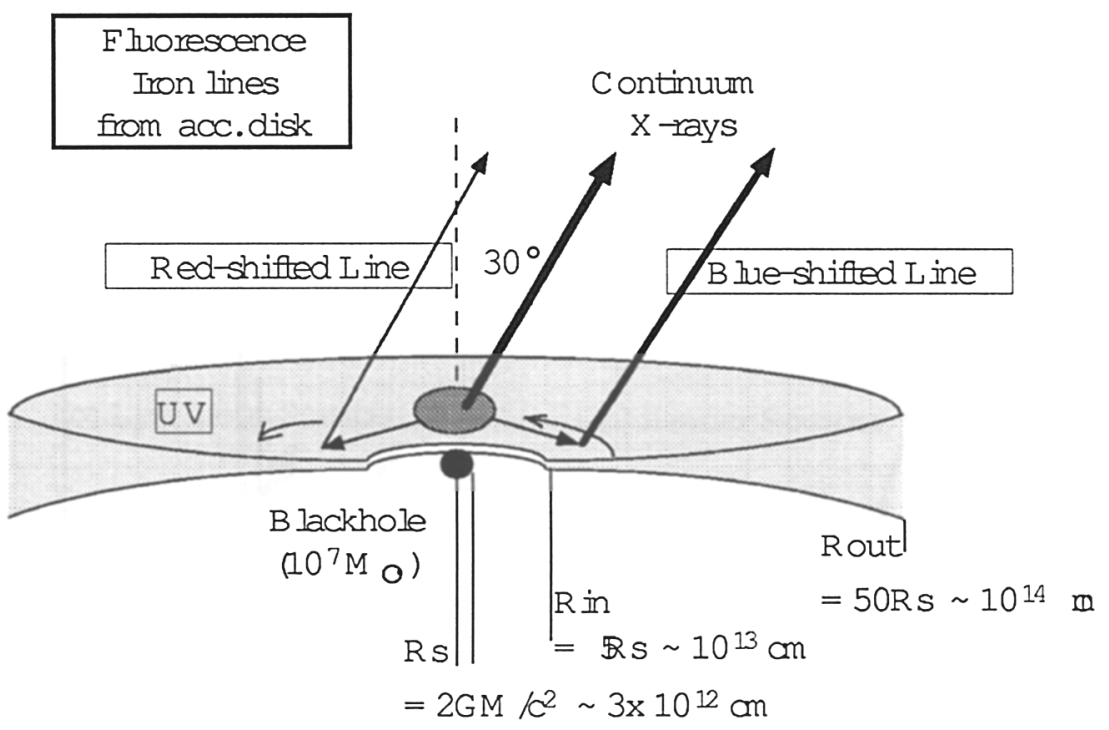

Figure 3. Distortion of the fluorescence iron- $\mathrm{K}$ lines from accretion disk.

will give a narrower profile. Such a situation can be realized by the height of the illuminating source, for example. An illuminating source at a higher position illuminates outer radii more (Figure $5 \mathrm{~d}$ ).

Reverberation Mapping Thus, we are able to examine the geometry of the central vicinity of AGN with the iron-K line profile as a probe. This approach will be enhanced by the future powerful missions. For example, the fast X-ray telescopes of XMM and Astro-E will allow us to perform reverberation mapping to get the absolute size of the disk and, finally, the mass of the black hole.

The innermost part will give the hatched part of the profile in Figure 6, while the outer part will contribute the dark part. Once the increase of the flux of the central source occurs, the hatched part becomes brighter first, and later the outer part will be enhanced. The time delay is the radial distance divided by the light velocity. Then, we will get the absolute value of $R_{\mathrm{s}}$. If the central mass is $10^{7}$ solar masses, a $15 R_{\mathrm{S}}$ difference give a time delay of $3000 \mathrm{sec}$.

\subsection{Photoionized Plasmas}

Warm Absorber Another phenomenon caused by the strong illumination is the existence of photoionized plasmas. The first example is the warm absorber. The low-energy absorption feature can be attributed to highly ionized plasmas. It was found by ROSAT. The ASCA CCD camera can determine the edge energies consistent to those of $\mathrm{O}$ VII and $\mathrm{O}$ VIII edges at $0.75-0.85 \mathrm{keV}$ (Fabian et al. 


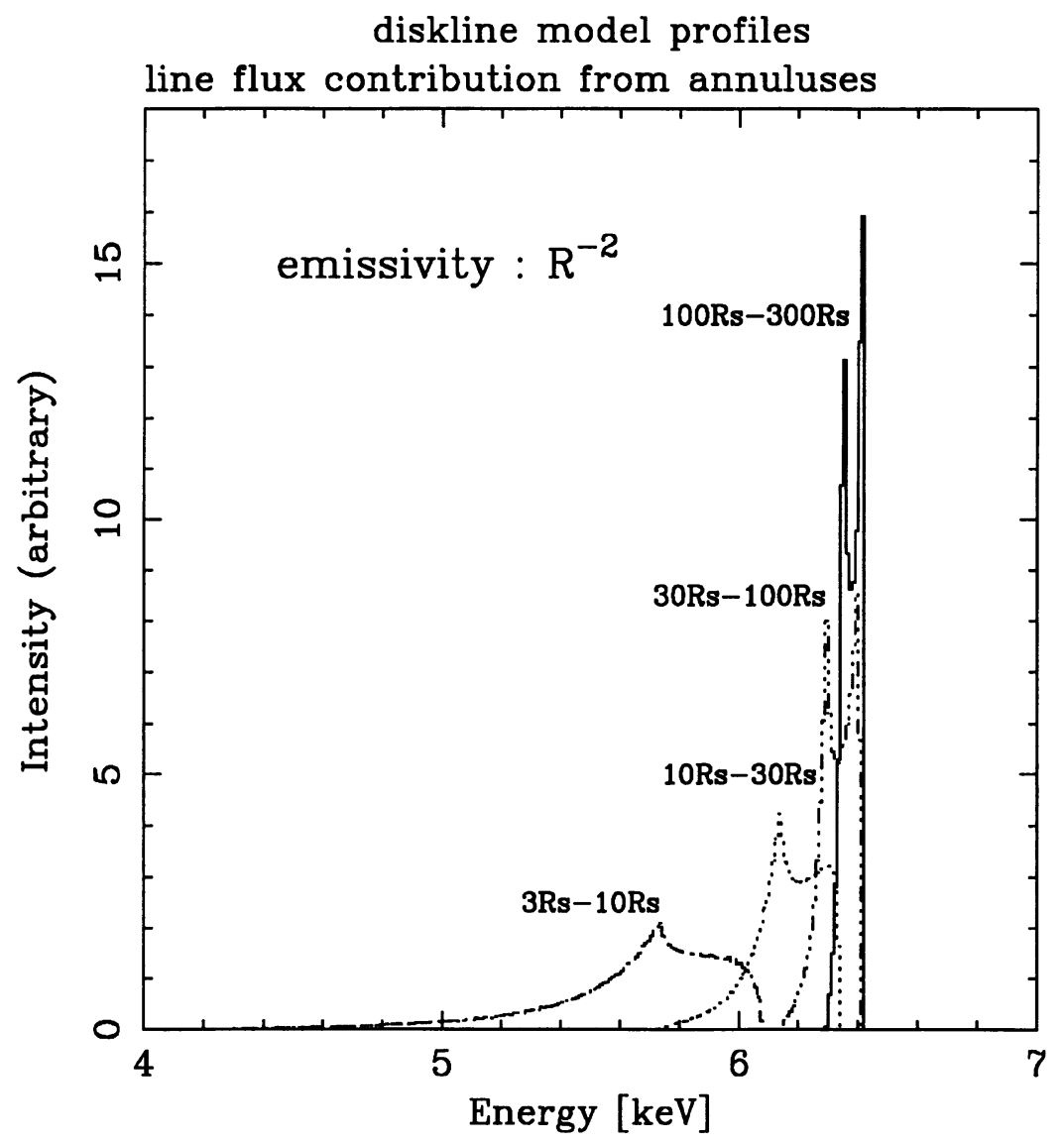

Figure 4. Model calculation of the iron-line profiles produced by different annuli of the illuminated accretion disk. Here, emissivity on the disk is assumed to be dependent on $R^{-2}$.

1994). Such absorption features can be attributed to the gas illuminated by the central source.

In the long-term observation of MCG-6-30-15, the change of the edge structure was found at the decrease of total flux. In the lower flux state, the O VIII edge became deeper, while O viI was almost constant (Otani et al. 1996). Another example of the edge profile change is found in NGC 5548. During the flux decrease of $40 \%$, the O VII depth increased, while the O VIII depth was constant.

Kallman \& McCray (1982) calculated the ionization state of elements in plasmas illuminated by high X-ray flux. In the brightest case, oxygen is fully ionized to O IX. When the source becomes fainter, O IX becomes O VIII, and part of $\mathrm{O}$ VIII is converted to $\mathrm{O}$ VII. Then, $\mathrm{O}$ vIII can be constant while $\mathrm{O}$ VII is doubled, which was seen in NGC 5548. 
(a) A verage Situaton

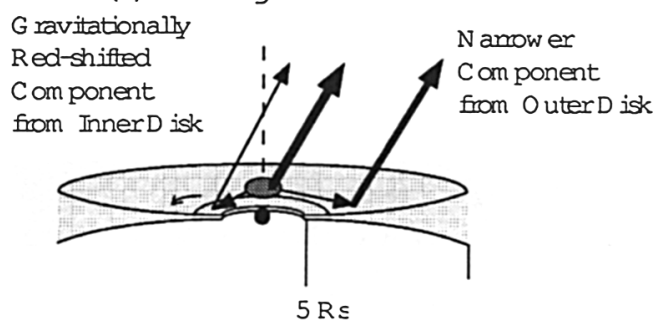

(b) Lum inous Sources

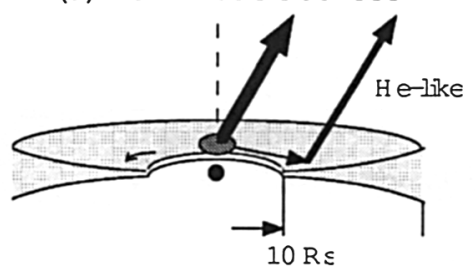

Lack of inner disk

$(R$ in $\rightarrow$ Large)

$===>$ Strong Thum ination by

the centralsouroe (c) Elare events

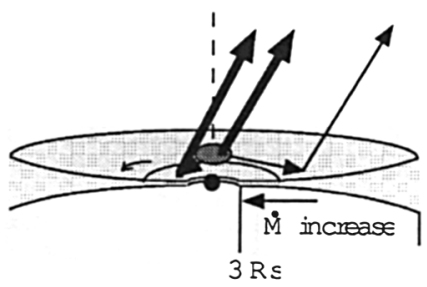

(d) H arder Sources

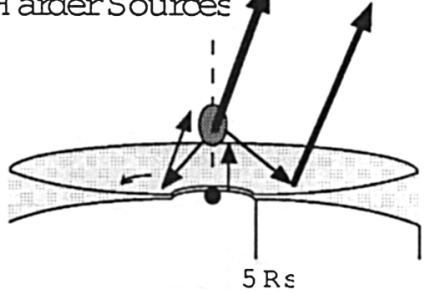

$M$ ore illum ination of outer disk

$(q \rightarrow$ zero)

$===>\mathrm{H}$ eight of the continuum souroe?

Figure 5. Accretion disks in different situations. (a) Typical accretion disk moderately illuminated. (b) Disk illuminated by strong radiation. (c) Temporal status of accretion disk at the time of flare. Accretion flow fills down to $3 R_{\mathrm{s}}$. (d) When the illuminating source is lifted above, illumination of the outer part of the disk is relatively high.

The most important, unknown parameter of the warm absorber is the location of the ionized gas. There is the trade off of the density $n$ and the distance $R$ to get the same ionization parameter $\xi\left(=L_{\mathrm{X}} / n R^{2}\right)$. If the definite distance and density were obtained, we would be able to answer the simple question whether the warm absorber can coexist with BRL clouds or not.

Seyfert II The second example of photoionized material is found from Seyfert IIs. In general, Seyfert II galaxies are favorable for examining the environment of the nucleus because the central bright source is blocked by the accretion tori, just as in a coronagraph.

Typical broadband spectra from Seyfert IIs are summarized by Turner et al. (1998). In the case of NGC 4507, a hard power-law component is seen with strong absorption with $n_{H}$ of $10^{23-24} \mathrm{H} / \mathrm{cm}^{2}$. Another soft component is seen below $2 \mathrm{keV}$ with some line features. In the case of $\mathrm{Mkn} 3$, the strongly absorbed component was found to be variable. In NGC 1068, almost no hard component is seen, but there are many emission lines from highly ionized elements. Even at the iron-K line complex, highly ionized iron- $\mathrm{K}$ lines are found. These strong iron 


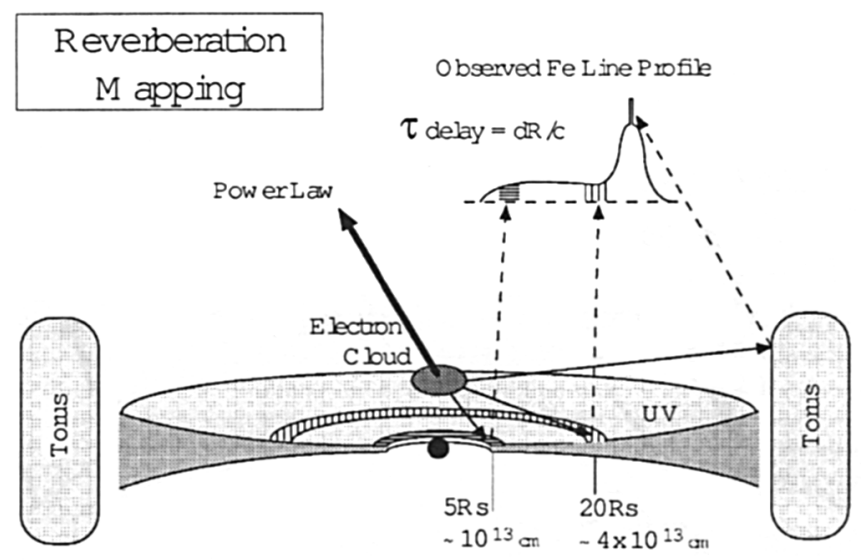

Figure 6. Reverberation mapping of the accretion disk by the line profile change.

lines suggest strong illumination and fluorescence, though the direct component from the center is completely obscured by the thick absorption torus.

Figure 7 is the correlation of the $\mathrm{Fe}$ emission-line equivalent width versus absorption column detected in low-energy X-rays. These galaxies produce $\mathrm{Fe}$ lines from the material consistent with the absorption column on the line of sight. However, these sources have stronger Fe lines, which suggests fluorescence by the material out of the line of sight.

\subsection{Link to Other Galaxies}

Starburst Galaxies versus Seyfert II Galaxies The high energy sensitivity of the ASCA telescope gives clues of the central activity of other galaxies, not only AGN but also normal galaxies and starburst galaxies. In order to distinguish the starburst galaxies from Sy II, clues from X-ray observations are used. Total $\mathrm{X}$-ray flux observed from starburst galaxies can be attributed to the hot plasma produced by the starburst activity. The iron-line equivalent width (EW) of starburst galaxies is relatively small, suggesting the small possibility of a strong illuminating source.

LINERs In the search for the central activity of LINERs (Low-Ionization $\mathrm{Nu}$ clear Emission Region galaxies) or normal galaxies, we detected a hard point source at the center of M106, where very high mass was found later to be within the immediate vicinity of an observed water maser. The energy spectrum ex- 


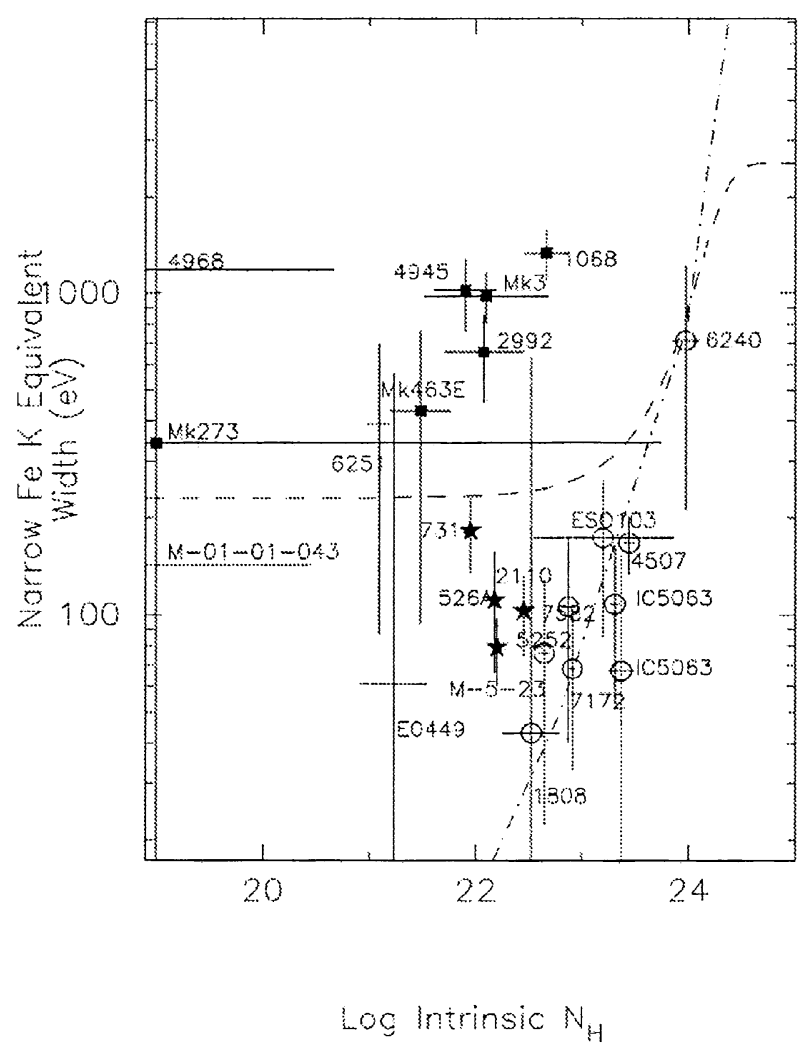

Figure 7. Iron-K line equivalent width from Seyfert IIs plotted against the absorption column density by Turner et al. 1997 .

hibits two components of a soft thermal source and a strongly absorbed hard component. If there are many such sources in LINERs and some normal galaxies, they may substantially contribute to the hard spectrum of the cosmic X-ray background. Harder sources like M106 are required to explain the power index of 1.4 for the CXB spectrum, which is inconsistent with the average power index of 1.7 from most Seyfert I galaxies.

Even without such a strongly absorbed core, the strong iron line is found in M51 to suggest strong central activity. In this case, the continuum spectrum and flux can be explained by the ambient, hot gas and the X-ray binaries in M51.

Our Galactic Center The nearest galactic nucleus is our Galactic center. ASCA observations of the Sgr region provide us with the distribution of 6.4 and $6.7 \mathrm{keV}$ 


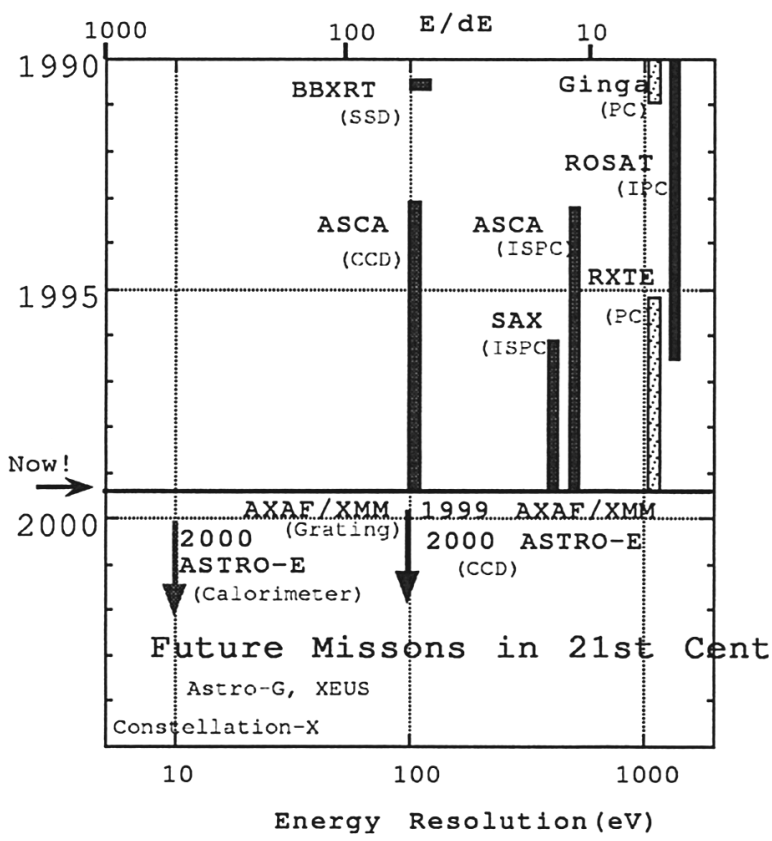

Figure 8. Energy resolution $E / \mathrm{d} E$ of current and future X-ray missions in a chronological table. (Astro-E should be listed at 2004-2005 instead of 2000.)

intensity. The spectrum of the real Galactic center exhibits both 6.4 and $6.7 \mathrm{keV}$ lines, while the Sgr B region is dominated by $6.4 \mathrm{keV}$ lines. Such a strong iron$\mathrm{K}$ line at $6.4 \mathrm{keV}$ suggests the illumination of cold material by a bright central source-maybe at Sgr A. The light travel time between Sgr A and B implies that the Galactic center could have been active several hundred years ago.

\section{Future Prospects}

Between July 1999 and February 2000, we will have three big missions. AXAF (Chandra) was launched in July 1999. It is equipped with an extremely highspatial-resolution telescope. XMM, an ESA mission, will be launched in December 1999 with high-throughput telescopes and CCD cameras. In 2004-2005, the Japan and US collaborative mission Astro-E is expected to be relaunched. It has the microcalorimeter on the focal plane to get an energy resolution $E / \mathrm{d} E$ of 600; that is ten times better than the ASCA CCD. In Figure 8, the energy resolutions $E / \mathrm{d} E$ of various missions are summarized. With those missions, we can perform high-resolution spectroscopy and fast Fe spectroscopy, which may allow us to perform reverberation mapping of the broad iron line. Figure 9 is a plot of the effective area of spectrometers onboard future missions. 
Future spectrometers

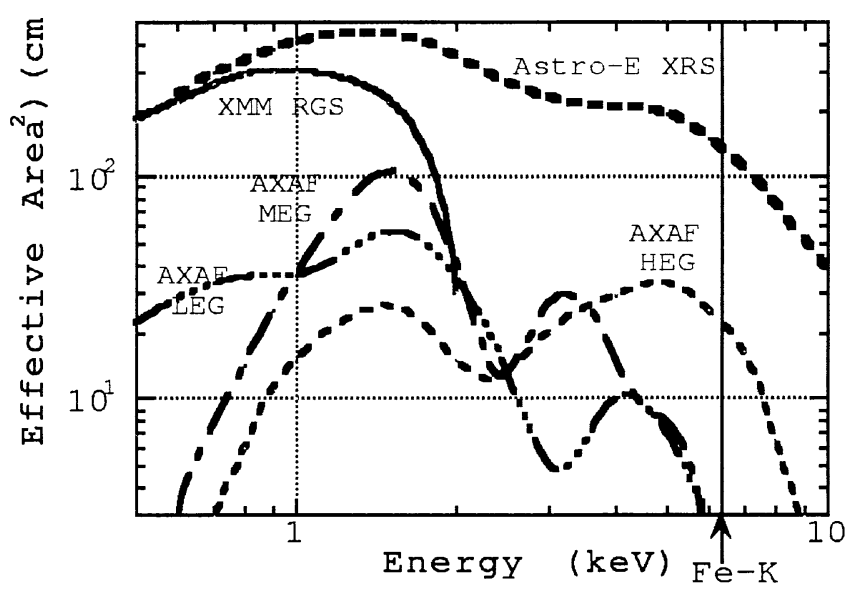

Figure 9. Effective area of high-resolution spectrometers for future missions.

\section{References}

Fabian, A., et al. 1994, PASJ, 46, L59

Iwasawa, K., Fabian, A. C., Young, A. J., Inoue, H., \& Matsumoto, C. 1999, MNRAS, 306, L19

Kallman, T. R., \& McCray, R. 1982, ApJS, 50, 263

Misaki, K., et al. 2000, ApJ, submitted

Nandra, K., George, I. M., Mushotzky, R. F., Turner, T. J., \& Yaqoob, T. 1997, ApJ, 488, L91

Nandra, K., \& Pounds, K. 1993, MNRAS, 268, 405

Otani, C., et al. 1996, PASJ, 48, 211

Tanaka, Y., et al. 1995, Nature, 375, 659

Turner, T. J., George, I. M., Nandra, K., \& Mushotzky, R. F. 1998, ApJ, 493, 91 